\title{
Efecto de diferentes sustratos en la calidad de plántulas de berenjena (Solanum melongena L.)
}

\section{Effects of different substrates on the quality of eggplant (Solanum melongena L.) seedlings}
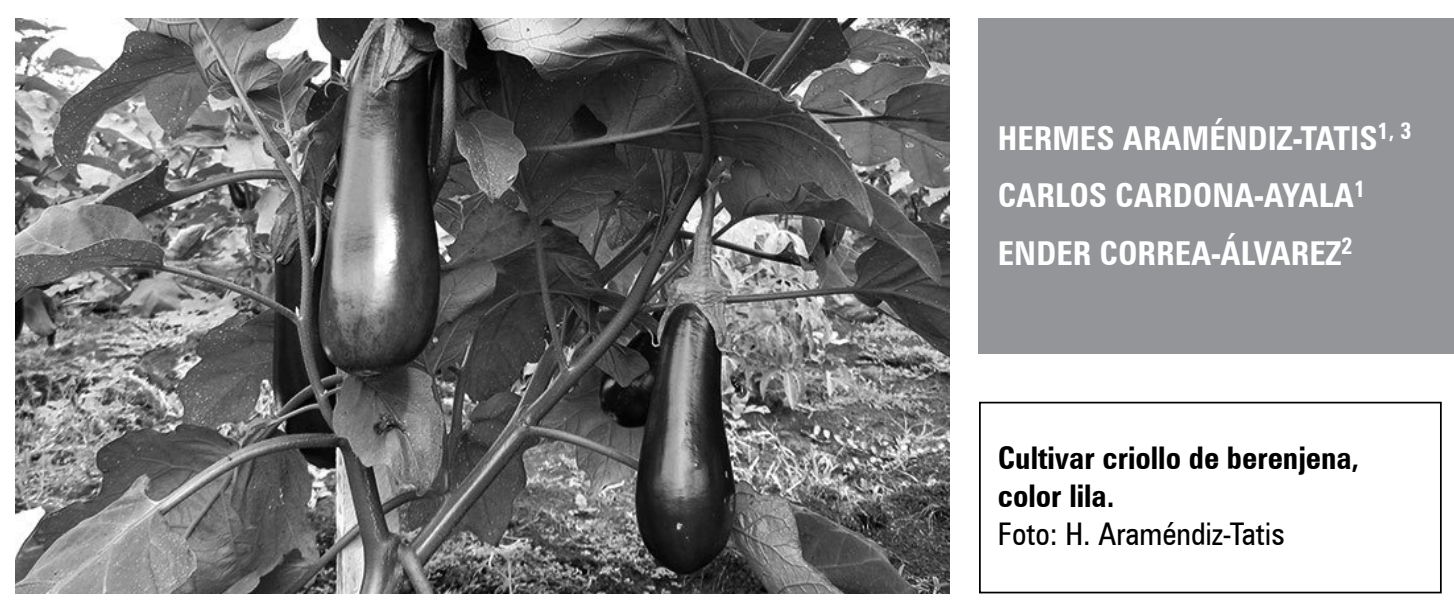

\section{RESUMEN}

La berenjena es una hortaliza con alta demanda en el departamento de Córdoba (Colombia), donde tiene importancia social y económica. El sistema actual de producción presenta algunos limitantes tecnológicos, uno de ellos es la producción de plántulas, y la de calidad de los sustratos. Con el fin de evaluar los sustratos disponibles de la región, se realizó un diseño completamente al azar con siete tratamientos, correspondientes a mezclas de sustratos (T1 $=50 \%$ aluvión $+50 \%$ casacarilla de arroz; T2 $=40 \%$ aluvión $+40 \%$ cascarilla de arroz $+20 \%$ lombriabono; T3 $=40 \%$ aluvión $+40 \%$ cascarilla de arroz $+20 \%$ gallinaza; T4 $=40 \%$ arena $+40 \%$ aluvión $+20 \%$ lombriabono; T5 $=40 \%$ arena $+40 \%$ aluvión $+20 \%$ gallinaza; $\mathrm{T} 6=40 \%$ aluvión $+40 \%$ fibra de coco $+20 \%$ lombriabono y T7 $=40 \%$ aluvión $+40 \%$ fibra de coco $+20 \%$ gallinaza), con seis repeticiones. Se utilizaron bolsas de polipropileno de 6 x 8 pulgadas y semillas del cultivar CO-029. Los sustratos constituidos por mezcla de lombriabono o gallinaza, arena y aluvión obtuvieron plántulas de mayor tamaño y óptima calidad; mientras que la cascarilla de arroz o fibra de coco, generaron plántulas de inferior calidad agronómica. El porcentaje de germinación y el índice de velocidad de germinación no fueron afectados por la composición de los sustratos.

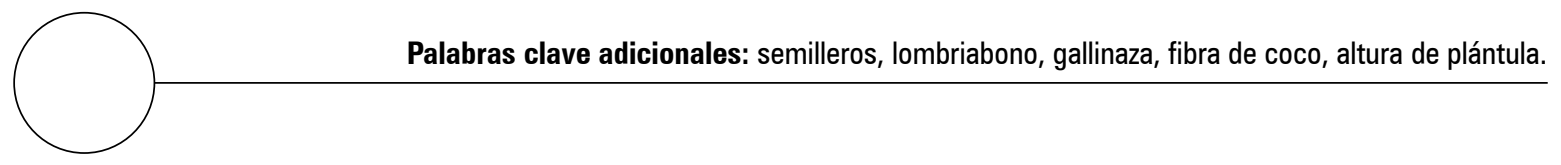

Facultad de Ciencias Agrícolas, Universidad de Córdoba, Montería (Colombia).

Corporación Colombiana de Investigación Agropecuaria (Corpoica), C.I. Caribia, Sevilla (Colombia).

Autor para correspondencia. haramendiz@hotmail.com 


\section{ABSTRACT}

The eggplant is a vegetable with high demand in the department of Córdoba (Colombia), where it has social and economic importance. The current production system has some technological constraints, including seedling production without quality control of the substrates, with negative effects on production, yield and fruit quality. In order to evaluate alternatives amongst the substrates available in the region, an experiment was carried out under a completely randomized design with seven treatments for mixtures of substrates (T1 $=50 \%$ alluvium $+50 \%$ rice husk; T2 $=40 \%$ alluvium $+40 \%$ rice husk $+20 \%$ vermicompost; T3 $=40 \%$ alluvium $+40 \%$ rice husk $+20 \%$ poultry manure; $\mathrm{T} 4=40 \%$ sand $+40 \%$ alluvium $+20 \%$ vermicompost; $\mathrm{T} 5=40 \%$ sand $+40 \%$ alluvium $+20 \%$ poultry manure; $\mathrm{T} 6=40 \%$ alluvium $+40 \%$ coconut fiber $+20 \%$ vermicompost y $\mathrm{T} 7=40 \%$ alluvium $+40 \%$ coconut fiber $+20 \%$ poultry manure), with six repetitions. The present study used $6 \times 8$ inch polypropylene bags and CO-029 cultivar seeds. The results indicate that larger seedlings and optimal quality were obtained with substrates consisting of a mixture of vermicompost and/or poultry manure, alluvial soil and sand; while rice husk and/or coconut fiber resulted in inferior agronomic quality seedlings. The percentage of germination and germination velocity were not affected by the composition of the substrates.

Additional key words: seedbeds, vermicompost, poultry manure, coconut fiber, seedling height.

La berenjena (Solanum melongena L.) es la solanácea de mayor importancia económica y social en el departamento de Córdoba, después del ají (Capsicum spp.); es originaria de Asía y forma parte de los hábitos alimentarios y culturales de dicho departamento; su introducción se atribuye a los árabes en el siglo pasado (Araméndiz et al., 2008).

El departamento de Córdoba es el principal productor de esta especie hortícola, a pesar de los limitantes tecnológicos, desde 2002 se redujeron los volúmenes de producción a una tasa anual del $6,70 \%$ y los rendimientos a 7,64\% (Agronet, 2013). Una de las razones de la disminución acelerada de los rendimientos por unidad de superficie, se sustenta en la ausencia de programas de certificación de semillas y plántulas; ya que los agricultores usan semillas derivadas de sus propios cultivos y producen plántulas con sustratos de desconocida calidad, además el número de semillas $/ \mathrm{m}^{2}$, la humedad, la infraestructura inapropiada y la presencia de problemas fitosanitarios ocasionan pérdidas hasta del $50 \%$ en las plantaciones (Velásquez y Amador, 2007).

El uso de los residuos de la agroindustria regional como materia prima para sustratos, representa una alternativa amigable con el ambiente y contribuye a la reducción de costos, como lo sostienen Sampaio et al. (2008). Así mismo, es importante considerar que un buen almácigo debe proporcionar precocidad y homogeneidad del cultivo, manejo eficiente de la semilla y la oportunidad de seleccionar plántulas mucho más vigorosas para su siembra en campo (Quesada y Méndez, 2005). Ello exige, que los sustratos posean buena capacidad de intercambio catió- 
nico, estabilidad física, esterilidad biológica, $\mathrm{pH}$ adecuado, capacidad de retención de agua y buen drenaje (Oliveira y Hernández, 2008).

En la producción de hortalizas de excelente calidad, la producción de plántulas es una de las etapas relevantes en el ciclo del cultivo, ya que tiene significativa influencia en las respuestas de la planta, desde el punto de vista nutricional y productivo, dado que existe una relación directa entre plántulas sanas y vigorosas y la producción en campo (Guimarães et al., 2002).

Moreira et al. (2010) evaluaron diferentes sustratos y dosis de fertilizantes para la producción de plántulas de berenjena de óptima calidad, donde el polvo de coco asociado con dosis de 16,30 a 20,41 g de fertilizantes, permitió una mayor producción de materia fresca y seca tanto de la parte aérea como de la radicular.

Considerando que la germinación de las semillas involucra una serie de eventos metabólicos, que tributan a la obtención de una plántula y que esta depende a su vez de la calidad del sustrato, el objetivo de la investigación fue evaluar diferentes tipos de sustratos sobre la producción de plántulas de berenjena de óptima calidad para su trasplante.

\section{MATERIALES Y MÉTODOS}

Localización. El estudio se realizó en el vivero de la Facultad de Ciencias Agrícolas de la Universidad de Córdoba, Montería, la cual está ubicada en el km 3 vía Cereté, zona media del Valle del Sinú, situada a $8^{\circ} 47^{\prime} \mathrm{N}$ y $75^{\circ} 51^{\prime} \mathrm{W}$, con una altitud de $15 \mathrm{msnm}$. La zona ecológica corresponde al bosque seco tropical con temperatura promedio de $28,8^{\circ} \mathrm{C}$, velocidad del viento media anual de 2,9 $\mathrm{km} \mathrm{h}^{-1}$, humedad relativa media del $77,4 \%$ y precipitación anual de 666,94 mm (TuTiempo, 2013).

Obtención de los sustratos. Los sustratos utilizados fueron aluvión cernido en tamiz de $2 \mathrm{~mm}$, cascarilla de arroz derivada de la agroindustria regional, fibra de coco no industrializada; lombriabono obtenido por el proceso de digestión de lombriz californiana, arena de río y gallinaza, la cual fue previamente fermentada para reducir los riesgos de bacterias y amoníaco.

Diseño experimental. Se utilizó un diseño completamente al azar con siete tratamientos (tabla 1) y seis repeticiones. Cada unidad experimental estuvo conformada por 10 bolsas de polipropileno de $6 \times 8$ pulgadas, sin fuelle, llenas con 1.300 $\mathrm{cm}^{3}$ de sustrato y sembradas con semillas del cultivar comercial CO-029.

Manejo en vivero. El local de propagación fue cubierto con polisombra de $80 \%$; se aplicó al sustrato de cada tratamiento $3 \mathrm{~g} \mathrm{~L}^{-1}$ de Trichoderma (Aramendiz et al., 2008), el crecimiento y desarrollo de las plantas fue monitoreado adecuadamente con la finalidad de mantenerlas libres de artrópodos, plagas y enfermedades.

Variables dependientes y registro de datos. Las variables de respuesta fueron: porcentaje de germinación e índice de velocidad de germinación, a través del conteo de plántulas normales hasta los 8 d según Maguire (1962), altura de plántulas $(\mathrm{mm})$, número de hojas verdaderas, longitud de la raíz $(\mathrm{mm})$, diámetro del tallo $(\mathrm{mm})$, peso fresco de la parte aérea (g), peso fresco de raíces (g), peso seco de la parte aérea (g) y peso seco de raíces (g) a los 15 d después de la germinación, sobre la base de cinco plántulas al azar.

Para la medición del peso fresco y seco de las partes aérea y radical de las plántulas, se utilizó una balanza analítica. El peso seco se determinó después de deshidratar las muestras a una temperatura de $70^{\circ} \mathrm{C}$, durante $72 \mathrm{~h}$ (Dias et al., 2008). Las medidas directas se hicieron por conteo y regla milimetrada, el diámetro del tallo se midió con un nonio, a una altura de $2 \mathrm{~cm}$ de la base del tallo.

Métodos estadísticos. Para las variables altura de planta y diámetro del tallo, se realizó un 
Tabla 1. Tratamientos evaluados en la obtención de plántulas de berenjena de óptima calidad.

\begin{tabular}{|l|c|c|c|c|c|c|}
\hline \multicolumn{1}{|c|}{ Sustratos } & Ar (\%) & Al (\%) & CA (\%) & FC (\%) & L (\%) & G (\%) \\
\hline Al50-CA50 & & 50 & 50 & & & \\
\hline Al40-CA40-L20 & & 40 & 40 & & 20 & \\
\hline Al40-CA40-G20 & & 40 & 40 & & & 20 \\
\hline Ar40-Al40-L20 & 40 & 40 & & & 20 & \\
\hline Ar40-Al40-G20 & 40 & 40 & & & & 20 \\
\hline Al40-FC40-L20 & & 40 & & 40 & 20 & \\
\hline Al40-FC40-G20 & & 40 & & 40 & & 20 \\
\hline
\end{tabular}

$\mathrm{Ar}=$ arena; $\mathrm{Al}=$ aluvión; $\mathrm{CA}=$ cascarilla de arroz; $\mathrm{FC}=$ fibra de $\operatorname{coco} ; \mathrm{L}=$ lombriabono; $\mathrm{G}=$ gallinaza.

análisis de varianza con los datos originales, utilizando el software SAS versión 9.2. Para el resto de variables, para el análisis de varianza se utilizó la transformación raíz cuadrada de $\mathrm{x}+$ 1 , con el fin de verificar de los supuestos, en términos de homogeneidad y distribución normal. La prueba de separación de medias de Tukey se utilizó al nivel 5\%.

\section{RESULTADOS Y DISCUSIÓN}

El análisis de varianza para las variables índice de velocidad de germinación y porcentaje de germinación no presentó significancia (tabla 2), lo que demostró que los sustratos no afectaron estos parámetros, debido a la calidad fisiológica de las semillas, que bajo las condiciones adecuadas de temperatura, oxígeno y humedad, no tuvieron dificultad para que ocurrieran las reacciones metabólicas necesarias para una germinación normal, como lo indican Silveira et al. (2002), Bezerra et al. (2009) y Reis et al. (2012).

Hubo efecto significativo para el número de hojas, hecho que significa mejores respuestas en los tratamientos Ar40-A140-L20 y Ar40-A140-G20, con 2,76 y 2,53 hojas/plántula, respectivamente (tabla 2), suficientes para el trasplante. La composición de estos dos sustratos que además del aluvión, contienen lombriabono y gallinaza, evidenciaron un mayor aporte nutricional y buena aireación que favoreció una mayor longitud de raíces (tabla 3), a causa de la presencia de promotores de crecimiento como Azotobacter, mejor anclaje y funcionamiento radicular, para la toma de agua, nutrientes y oxígeno, lo que favorece la actividad fotosintética y el transporte de asimilados (Taiz y Zeiger, 2004; Ortega-Martínez et al., 2010).

La presencia de un mayor número de hojas de acuerdo con Moreira et al. (2010), es de gran relevancia, ya que constituyen una de las principales fuentes de fotoasimilados (azúcares, hormonas, aminoácidos, etc.) y nutrientes para la adaptación de la plántula al sitio definitivo durante el primer mes. Por otra parte, en los tratamientos A150-CA50, A140-CA40-L20, A140-CA40-G20, A140-FC40-L20 y A140-FC40-G20, la presencia de fibra de coco y cascarilla de arroz por su bajo contenido nutricional (Schmitz et al., 2002; Costa et al., 2007), constituye una desventaja para el desarrollo radicular, dado que en condiciones de estrés los fotoasimilados son orientados hacia las raíces en detrimento de la parte aérea (Samôr et al., 2002), y con ello, la formación de plántulas de óptima calidad se ve afectada (Silveira et al., 2002) lo que conlleva a realizar procesos de fertirrigación para producir plántulas de buena calidad (Bezerra et al., 2009).

La altura de plántulas registró diferencias significativas entre tratamientos, donde los sustratos Ar40-A140-L20 y Ar40-A140-G20, presentaron los mayores valores con 53,66 y $51,56 \mathrm{~mm}$, res- 
Tabla 2. Valores medios de índice de velocidad de germinación (IVG), porcentaje de germinación (G), número de hojas (NH), altura de plántula (AL) y diámetro del tallo (DT).

\begin{tabular}{|l|c|c|c|c|c|}
\hline \multicolumn{1}{|c|}{ Sustratos $^{1}$} & $\begin{array}{c}\text { IVG } \\
\text { (plántulas/día) }\end{array}$ & $\begin{array}{c}\mathrm{G} \\
(\%)\end{array}$ & $\begin{array}{c}\mathrm{NH} \\
(\mathrm{No})\end{array}$ & $\begin{array}{c}\text { AL 15 } \mathrm{d} \\
(\mathrm{mm})\end{array}$ & $\begin{array}{c}\text { DT } 15 \mathrm{~d} \\
(\mathrm{~mm})\end{array}$ \\
\hline Al50-CA50 & $1,38 \mathrm{a}$ & $93,33 \mathrm{a}$ & $1,00 \mathrm{c}$ & $24,35 \mathrm{~b}$ & $0,90 \mathrm{~b}$ \\
\hline Al40-CA40-L20 & $1,51 \mathrm{a}$ & $96,67 \mathrm{a}$ & $1,00 \mathrm{c}$ & $25,40 \mathrm{~b}$ & $0,82 \mathrm{~b}$ \\
\hline Al40-CA40-G20 & $1,29 \mathrm{a}$ & $78,33 \mathrm{a}$ & $1,53 \mathrm{bc}$ & $27,96 \mathrm{~b}$ & $0,87 \mathrm{~b}$ \\
\hline Ar40-Al40-L20 & $1,77 \mathrm{a}$ & $98,33 \mathrm{a}$ & $2,76 \mathrm{a}$ & $53,66 \mathrm{a}$ & $1,37 \mathrm{a}$ \\
\hline Ar40-AI40-G20 & $1,62 \mathrm{a}$ & $98,33 \mathrm{a}$ & $2,53 \mathrm{a}$ & $51,56 \mathrm{a}$ & $1,41 \mathrm{~b}$ \\
\hline Al40-FC40-L20 & $1,51 \mathrm{a}$ & $91,67 \mathrm{a}$ & $1,76 \mathrm{~b}$ & $30,69 \mathrm{~b}$ & $0,94 \mathrm{~b}$ \\
\hline Al40-FC40-G20 & $1,61 \mathrm{a}$ & $98,33 \mathrm{a}$ & $1,08 \mathrm{bc}$ & $34,73 \mathrm{~b}$ & $1,01 \mathrm{~b}$ \\
\hline C.V. (\%) & 8,10 & 14,60 & 8,50 & 20,18 & 16,57 \\
\hline
\end{tabular}

${ }^{1}$ Abreviaturas ver tabla 1.

Promedios con letras distintas indican diferencia significativa según la prueba de Tukey $(P \leq 0,05)$.

Tabla 3. Valores medios de peso fresco parte aérea (PFA), peso seco parte aérea (PSA), peso fresco de raíces (PFR), peso seco de raíces (PSR) y longitud de raíces (LR).

\begin{tabular}{|l|c|c|c|c|c|}
\hline \multicolumn{1}{|c|}{ Sustratos } & $\begin{array}{c}\text { PFA } \\
(\mathrm{g})\end{array}$ & $\begin{array}{c}\text { PSA } \\
(\mathrm{g})\end{array}$ & $\begin{array}{c}\text { PFR } \\
(\mathrm{g})\end{array}$ & $\begin{array}{c}\text { PSR } \\
(\mathrm{g})\end{array}$ & $\begin{array}{c}\text { LR } \\
(\mathrm{mm})\end{array}$ \\
\hline Al50-CA50 & $0,05 \mathrm{~b}$ & $0,005 \mathrm{~b}$ & $0,02 \mathrm{~b}$ & $0,003 \mathrm{~b}$ & $58,70 \mathrm{~b}$ \\
\hline Al40-CA40-L20 & $0,06 \mathrm{~b}$ & $0,006 \mathrm{~b}$ & $0,03 \mathrm{~b}$ & $0,004 \mathrm{~b}$ & $73,90 \mathrm{~b}$ \\
\hline Al40-CA40-G20 & $0,15 \mathrm{~b}$ & $0,011 \mathrm{~b}$ & $0,04 \mathrm{~b}$ & $0,004 \mathrm{~b}$ & $67,50 \mathrm{~b}$ \\
\hline Ar40-Al40-L20 & $0,59 \mathrm{a}$ & $0,041 \mathrm{a}$ & $0,06 \mathrm{~b}$ & $0,005 \mathrm{~b}$ & $92,57 \mathrm{ab}$ \\
\hline Ar40-AI40-G20 & $0,70 \mathrm{a}$ & $0,053 \mathrm{a}$ & $0,14 \mathrm{a}$ & $0,011 \mathrm{a}$ & $122,67 \mathrm{a}$ \\
\hline Al40-FC40-L20 & $0,18 \mathrm{~b}$ & $0,013 \mathrm{~b}$ & $0,03 \mathrm{~b}$ & $0,004 \mathrm{~b}$ & $64,33 \mathrm{~b}$ \\
\hline Al40-FC40-G20 & $0,09 \mathrm{~b}$ & $0,007 \mathrm{~b}$ & $0,03 \mathrm{~b}$ & $0,003 \mathrm{~b}$ & $72,67 \mathrm{~b}$ \\
\hline C.V. (\%) & 4,2 & 0,4 & 1,4 & 0,1 & 16,8 \\
\hline
\end{tabular}

${ }^{1}$ Abreviaturas ver tabla 1.

Promedios con letras distintas indican diferencia significativa según la prueba de Tukey $(P \leq 0,05)$.

pectivamente. Los demás tratamientos mostraron alturas entre 24,35 y $34,73 \mathrm{~mm}$ (tabla 2 ); ello obedece a que las plántulas cuyas raíces poseen mayor longitud aprovechan mejor las condiciones físicas y nutricionales aportadas por los sustratos, especialmente el fósforo por su papel en el crecimiento de las plántulas, ya que forma parte del ATP, responsable del almacenamiento y transporte de energía para procesos de sínte- sis de compuestos orgánicos y absorción activa de nutrientes (Oliveira y Hernández, 2008; Rodrigues et al., 2010). Aquellos sustratos asociados con la presencia de cascarilla de arroz y fibra de coco, realizan poco aporte nutricional en cuanto a nitrógeno y fósforo, para un buen desarrollo de plántulas (Sampaio et al., 2008); lo que es corroborado por Arenas et al. (2002) al reportar en tomate, plántulas de menor tamaño 
con sustratos compuestos con un 50\% de fibra de coco, en tanto que Andrade-Rodríguez et al. (2008), en papaya, reportaron menor altura de plántula con este mismo sustrato, a causa de la clorosis general por la liberación de fenoles.

El diámetro del tallo registró respuesta similar a la de la altura de plántula, donde los sustratos Ar40-A140-L20 y Ar40-A140-G20, registraron los mayores diámetros de tallo con 1,37 y 1,41 $\mathrm{mm}$, respectivamente, superando en más de 0,40 $\mathrm{mm}$ a los demás sustratos (tabla 2). Esta superioridad en Ar40-A140-L20 y Ar40-A140-G20, con respecto al resto de sustratos, obedece a un mayor aporte nutricional de los mismos. Es sabido que los reducidos niveles de nitrógeno y fósforo limitan la producción de plántulas agronómicamente superiores, puesto que el funcionamiento de las raíces está directamente ligado a las condiciones de aireación, contenido de agua y disponibilidad de los nutrientes (Andrade-Rodríguez et al., 2008). En este sentido, Quesada y Méndez (2005), después de evaluar el diámetro del tallo en tomate, pepino, lechuga y brócoli, concluyeron que el lombriabono con abono orgánico favoreció mucho más el desarrollo del tallo, tanto por su aporte nutricional como por sus propiedades físicas; en aquellos con presencia de fibra de coco, hubo escasa influencia en el desarrollo radicular.

Los valores del peso fresco y seco tanto de la parte aérea como radicular manifestaron la misma tendencia que la altura de plántula y diámetro del tallo. Los valores más altos de estas variables correspondieron a los sustratos Ar40-A140-L20 y Ar40-A140-G20 (tabla 3), que a su vez presenta- ron mayor número de hojas por planta y longitud de raíces (tablas 2 y 3). El bajo peso aéreo y radicular, tanto fresco como seco del resto de los tratamientos, obedeció al pobre crecimiento y desarrollo alcanzado por la planta ante las limitaciones físicas y químicas de los sustratos, con desventajas agronómicas (Quesada y Méndez, 2005).

Se encontró una relación clara entre la longitud de las raíces, la altura de plántula, diámetro del tallo, peso fresco y seco de la parte aérea y peso fresco y seco de raíces, ya que los sustratos Ar40-A140-L20 y Ar40-A140-G20, lograron los mayores valores (tablas 2 y 3 ), lo que permite un mejor aprovechamiento del volumen del sustrato en cuanto a humedad, características físicas y químicas, reflejándose en una mejor calidad de plántula para su trasplante al sitio definitivo, hecho que coincide con los resultados reportados por Araújo et al. (2010) en papaya y Rodrigues et al. (2010) en tomate, quienes usaron sustratos que en su composición tenían un alto porcentaje de origen animal.

\section{CONCLUSIONES}

El desarrollo de plántulas de óptima calidad de berenjena fue favorecido por sustratos en cuya mezcla contenía lombriabono y/o gallinaza, asociado con arena de río.

Los sustratos con presencia de fibra de coco y/o cascarilla de arroz, obtuvieron plántulas con desventaja agronómica para su trasplante. 


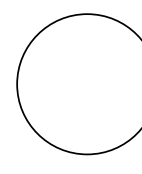

Agronet. 2013. Análisis estadístico de área y producción agrícola y pecuaria. En http://www.agronet.gov. co/agronetweb1/Estadísticas/ReportesEstadísticos. aspx; consulta: febrero 16 de 2013.

Andrade-Rodríguez, M., J.J. Ayala-Hernández, I. AliaTejacal, H. Rodríguez-Mendoza, C.M. Acosta-Durán y V. López-Martínez. 2008. Efecto de promotores de la germinación y sustratos en el desarrollo de plántulas de papayo. Rev. Fac. Agron. (LUZ) 25 (4), 617-625.

Araméndiz, H., C. Cardona, A. Jarma y M. Espitia. 2008. El cultivo de la berenjena (Solanum melongena L.). Editorial Produmedios, Bogotá.

Araújo, W.B.M., R.D. Alencar, V. Mendonça, E.V. Medeiros, R.C. Andrade y R.R. Araujo. 2010. Esterco caprino na composição de substratos para formação de mudas de mamoeiro. Ciênc. Agrotec. 34(1), 6873.

Arenas, M., C.S. Vavrina, J.A. Cornell, E.A. Hanton y G.J. Hochmuth. 2002. Coir as an alternative to peat in media for tomato transplant production. HortScience 37 (2), 309-312.

Bezerra, F.C., F.V. Meyer Ferreira y T.C. Silva. 2009. Produção de mudas de berinjela em substratos à base de resíduos orgânicos e irrigadas com água ou solução nutritiva. Hortic. Bras. 27 (2), 1348-1352.

Costa, C.A., S.J. Ramos, R.A. Sampaio, D.O. Guilherme y L.A. Fernandes. 2007. Fibra de coco e resíduo de algodão para substrato de mudas de tomateiro. Hortic. Bras. 25 (4), 387-391

Dias, M.A., J.C. Lopes, N.B. Corrêa y D.C. Dias. 2008. Germinação de sementes e desenvolvimento de plantas de pimenta malagueta em função do substrato e da lâmina de água. Rev. Bras. Sementes 30 (3), 115-121.

Guimarães, V.F., M.M. Echer y K. Minami, 2002. Métodos de produção de mudas, distribuição de matéria seca produtividade de plântulas de beterraba. Hortic. Bras. 20 (3), 505-509.

Maguire, J.D. 1962. Speed of germination aid in selection and evaluation for seedling emergence and vigor. Crop Sci. 2(1), 176-177.

Moreira, M.A., F.M. Dantas, F.G. Bianchini y P.R. Viégas. 2010. Produção de mudas de berinjela com uso de pó de coco. Rev. Bras. Produtos Agroindustriais 12(2), 163-170.

\section{REFERENCIAS BIBLIOGRÁFICAS}

Oliveira, A.B. y F.F.F. Hernández, 2008. Absorção de nutrientes em mudas de berinjela cultivadas em substratos alternativos. Rev. Ciênc. Agron. 39(4), 583-589.

Ortega-Martínez, L.D., J. Sánchez-Olarte, R. Díaz-Ruiz y J. Ocampo-Mendoza. 2010. Efecto de diferentes sustratos en el crecimiento de plántulas de tomate (Lycopersicum esculentum Mill.). Ra Ximhai 6(3), 365-372.

Quesada, G.y R. Méndez. 2005. Evaluación de sustratos para almácigos de hortalizas. Agron. Mesoamericana 16(2), 171-183.

Reis, R., R.M. Guimarães, A.R. Vieira, N.R. Gonçalves y V. Costa. 2012. Physiological quality of osmoprimed eggplant seeds. Ciênc. Agrotec. 36(5), 526-532.

Rodrigues, E.T., P.A.M. Leal, E. Costa, E. de Paula y V. Gomes. 2010. Produção de mudas de tomateiro em diferentes substratos e recipientes em ambiente protegido. Hortic. Bras. 28(4), 483-488.

Samôr, O.J.M., J.G. de A. Carneiro, D.G. Barroso y P.S. dos S. Leles. 2002. Qualidade de mudas de angico e sesbânia, produzidas em diferentes recipientes e substratos. Rev. Árvore 26(2), 209-215.

Sampaio, R.A., S.J. Ramos, D.O. Guilherme, C.A. Costa y L.A. Fernandes. 2008. Produção de mudas de tomateiro em substratos contendo fibra de coco e pó de rocha. Hortic. Bras. 26 (4), 499-503.

Silveira, E.B.; V.J.L.B. Rodrigues, A.M.A. Gomes, R.L.R. Mariano y J.C.P. Mesquita. 2002. Pó de coco como substrato para produção de mudas de tomateiro. Hortic. Bras. 20 (2), 211-216.

Schmitz, J.A., P.V.D. Souza y A.N. Kämpf. 2002. Propriedades químicas e físicas de substratos de origem mineral e orgânica para o cultivo de mudas em recipientes. Ciência Rural 32 (6), 937-944.

Taiz, L. y E. Zeiger. 2004. Fisiología vegetal. $3^{a}$ ed. Editorial Armed, Porto Alegre, Brasil.

TuTiempo. 2013. Clima en Monteria / Los Garzones el tiempo en 2012. Datos reportados por la estación meteorológica: 800630 (SKMR). Valores climáticos medios y totales anuales. http://www.tutiempo. net/clima/Monteria_Los_Garzones/2012/800630. htm; consulta: abril de 2013 .

Velásquez, V.R. y M.D. Amador. 2007. Análisis sobre la investigación fitopatológica de Chile seco (Capsicum ornearum L.). Rev. Mex. Fitopatol. 25(1), 80-84. 